\title{
Obtenção de nanopartículas magnéticas utilizando materiais do cotidiano: síntese, caracterização e abordagem didática para o ensino médio
}

Obtaintion of magnetic nanoparticles using everyday materials: synthesis, characterization and didactic approach for high school

\author{
Andresa da Costa Ribeiro ${ }^{10}$, Marcia da Costa ${ }^{2}$, Cleciane Ramos dos Santos $^{1}$, \\ Taiana Gabriela Moretti Bonadio ${ }^{1}$, Tania Toyomi Tominaga*1 \\ ${ }^{1}$ Universidade Estadual do Centro-Oeste, Guarapuava, PR, Brasil. \\ ${ }^{2}$ Universidade Federal do Espírito Santo, Alegre, ES, Brasil.
}

Recebido em 03 de setembro de 2020. Revisado em 11 de novembro de 2020. Aceito em 16 de novembro de 2020.

Neste trabalho é apresentada uma prática experimental com materiais do cotidiano para obtenção de nanopartículas magnéticas e uma proposta didática com o tema Nanociências e Nanotecnologia, baseada na abordagem CTSA. As NPmag foram caracterizadas por espalhamento de luz e microscopia eletrônica de varredura. A proposta aliou a atividade experimental, discussões referentes à caracterização, introdução aos temas e suas aplicações. Os resultados da caracterização sugerem que a metodologia para obtenção das NPmag é eficiente para fins didáticos e a proposta didática proporcionou aprendizagem de conteúdos científicos e suas aplicações.

Palavras-chave: Física, Nanociência, Nanotecnologia, CTSA, Ensino Médio, Nanopartículas magnéticas.

In this work, an experimental practice is presented using low-cost materials, to obtain magnetic nanoparticles and also a didactic proposal was applied about the theme Nanoscience and Nanotechnology, based on a CTSA approach. The nanoparticles were characterized by light scattering and scanning electron microscopy. The proposal was composed of an experimental activity, discussions about the result of the characterization and introduced the themes Nanoscience and Nanotechnology and its applications. The results of the characterization suggest that the used methodology to obtain the nanoparticles was efficient for didactic purposes and the didactic proposal provided learning of scientific content and its applications.

Keywords: Physics; Nanoscience; Nanotechnology; STDE; High school; Magnetic Nanoparticles.

\section{Introdução}

Nos últimos anos os termos "Nanociência" e "Nanotecnologia" vêm ganhando grande notoriedade em diferentes esferas da sociedade. Isto porque esta tecnologia tem cada vez mais impactado na qualidade de vida das pessoas. Sua aplicação vai desde a área da saúde, no desenvolvimento de dispositivos e fármacos para tratamento de doenças, bem como apresentam grande aplicação nas áreas de estética, cosmética, alimentícia e ambiental [1]. Apesar da Nanotecnologia ser uma área da ciência reconhecida a menos de um século atrás, a melhora nas pesquisas e avanços tecnológicos alcançados, a tornaram atualmente um dos temas de maior interesse no mundo [2].

Pode-se definir nanociência como o ramo que estuda as estruturas atômicas e moleculares que possuem dimensões na escala nanométrica [2, ou seja, estruturas com tamanhos entre 0,1 e 100 nanômetros.

\footnotetext{
*Endereço de correspondência: ttominaga@unicentro.br
}

Um nanômetro, equivale a $10^{-9} \mathrm{~m}$ ou 1 bilionésimo de metro, algo 60000 vezes menor que a espessura média de um fio de cabelo 3, 4.

A manipulação e aplicação industrial de estruturas nanométricas recebe o nome de Nanotecnologia [2] que pode ser aplicada em diversas áreas do conhecimento [5], tais como na Física, Química, Biologia, Matemática, Computação, Engenharias, Ciências da saúde, e o meio ambiente [1, 2].

O uso de nanopartículas magnéticas pode ser eficiente em aplicações ambientais na despoluição de águas residuais [5]. Tais nanopartículas possuem baixo custo de fabricação e facilidade na separação por meio da utilização de campos magnéticos externos, devido à propriedade ferromagnética. Na área da saúde, estudos mostram que nanopartículas podem ser utilizadas no desenvolvimento de formulações nanotecnológicas destinadas ao tratamento de câncer 2, de doenças inflamatórias, cardiovasculares, neurológicas, dentre outras [5]. Além disso, a manipulação de materiais nanométricos pode dar origem a vários novos produtos, ou seja, as nanoestruturas 
podem estar presentes em cosméticos, displays, bolas de tênis, tinturas de para-choques de automóveis, tratamento de superfícies e até mesmo nos preservativos [6].

Para a evolução de uma sociedade mais crítica, informada, participativa e atuante, é importante que conhecimentos atuais, tais como os de nanociência e nanotecnologia, alcancem cada vez mais uma audiência não restrita à academia e à indústria. Uma estratégia que facilita a expansão desses temas é a aplicação de propostas educacionais acessíveis para jovens do Ensino Médio. No entanto, apesar da presença de apontamentos para o ensino destes ramos nas Orientações Curriculares para o Ensino Médio e também nas Diretrizes Nacionais para a Educação Básica, muito pouco se discute sobre o tema em sala de aula [1].

Na disciplina de Física, por exemplo, grande parte dos conteúdos abordados nas escolas da educação básica estão relacionados à Física Clássica, construída antes do século XX, com pouca ou nenhuma relação com o desenvolvimento tecnológico atual [2]. Além disso, o currículo visto nas escolas atualmente é formado por muitas disciplinas cujo saberes não estão sendo articulados [7], o que não favorece ao aluno a compreensão, contextualização e criticidade em relação ao que é estudado.

Uma proposta didática bastante utilizada para trabalhar o tema Nanociência e Nanotecnologia, principalmente nos ensinos de Química e Biologia, é o enfoque Ciência-Tecnologia-Sociedade-Ambiente (CTSA) [8-10]. Nestas abordagens, um dos eixos norteadores é a reflexão a respeito da interrelação entre ciência, tecnologia, sociedade e ambiente envolvida na compressão de temáticas científicas.

É inegável que o desenvolvimento científico e tecnológico tem ocasionado inúmeras mudanças na sociedade contemporânea e discutir a respeito dessas transformações deveria fazer parte da formação educacional dos indivíduos, uma vez que "o cidadão merece aprender a ler e entender - muito mais do que conceitos estanques - a ciência e a tecnologia, com suas implicações e consequências, para poder ser elemento participante nas decisões de ordem política e social que influenciarão o seu futuro e o dos seus filhos" [11.

As mudanças científico-tecnológicas são fatores que contribuem para modelar as formas de vida, dessa maneira deve-se buscar uma formação científica crítica para que as pessoas possam se relacionar criticamente com a ciência e a tecnologia (CT), bem como com todas as implicações sociais e ambientais a elas relacionadas [12.

No âmbito do enfoque CTSA (Ciência, Tecnologia, Sociedade e Ambiente), a formação de cidadãos aptos a refletirem e tomarem decisões a respeito de ciência e tecnologia deve ser contemplada no Ensino de Ciências. Neste sentido, uma das preocupações do ensino de Ciências, e aqui volta-se a atenção para o Ensino de Física, é a preparação dos indivíduos para a participação democrática em processos que envolvam ciência e tecnologia.
Santos e Mortimer [13] defendem que a abordagem CTSA, voltada para a educação, pode ser considerada como uma integração entre educação científica, tecnológica e social, em que os conteúdos científicos e tecnológicos são estudados juntamente com a discussão de seus aspectos históricos, éticos, políticos e socioeconômicos. O objetivo dessa abordagem no Ensino Médio é desenvolver a alfabetização científica e tecnológica dos cidadãos, auxiliando o aluno a construir conhecimentos, habilidades e valores necessários para tomar decisões responsáveis sobre questões de ciência e tecnologia na sociedade e atuar na solução de tais questões.

No entanto, vale ressaltar que defender a inclusão dos aspectos sociais e ambientais no ensino de Física não significa menor preocupação com o ensino de conceitos. $\mathrm{O}$ que muda com essa abordagem é que ela inclui temas relevantes para os alunos e os conteúdos e conceitos deixam de ter somente um significado futuro, para aqueles que vão seguir seus estudos em nível superior, para ter um significado atual, passam a constituir-se em meios, em ferramentas para a compreensão de problemáticas da sua realidade, dando significado a aprendizagem dos conceitos e, além disso, pode se tornar uma oportunidade de mudança de comportamento [14.

A ideia de levar para sala de aula o debate sobre as relações CTSA - tanto no Ensino Fundamental quanto no Ensino Médio, vem sendo difundida por meio dos Parâmetros Curriculares Nacionais (PCNs) como forma de Educação Tecnológica, que tem o objetivo de levar os alunos a compreender a dimensão social da ciência e tecnologia, tanto do ponto de vista dos seus antecedentes sociais, quanto de suas consequências sociais e ambientais e encontram respaldo em diversos objetivos, tanto da Lei de Diretrizes e Bases (LDB) quanto dos Parâmetros Curriculares Nacionais para o Ensino Médio (PCNEMs) e mais recentemente nas competências da (Base Nacional Comum Curricular) BNCC [15-17.

Como levar essa abordagem para a sala de aula? Uma das mudanças ao se usar este tipo de abordagem são destacadas por Auler [14] onde, ao invés do ensino ser pautado em conteúdos ele passa a ser pautado em temas. Temáticas que relacionam ciência, tecnologia, sociedade e ambiente e que, por natureza, também podem ser interdisciplinares. A abordagem de temas interdisciplinares é defendida como uma maneira de romper com as barreiras das ciências, favorecendo a integração entre diferentes ciências e oportunizando aos alunos uma articulação de saberes de diferentes disciplinas [18].

Ao se pensar no enfoque CTSA voltado ao ensino de Física, não há estudos significativos sobre os temas Nanociência e Nanotecnologia (N\&N). Grande parte do que já foi publicado refere-se a análises teóricas sobre a temática [19, 20. Sendo assim, pretende-se neste trabalho apresentar uma alternativa para abordar essa temática no currículo de Física do Ensino Médio. Essa alternativa consiste em uma proposta didática que envolve 
a obtenção de nanopartículas magnéticas no ambiente escolar utilizando materiais comerciais do cotidiano, tais como vinagre, palha de aço e água oxigenada bem como uma discussão sobre os temas abordados.

\section{Obtenção e Caracterização das Nanopartículas Magnéticas (NPmag)}

Para preparar NPmag vários métodos químicos têm sido desenvolvidos ao longo dos anos, tais como a coprecipitação, sol-gel, microemulsão e etc [21-24]. Para este trabalho a metodologia usada foi a co-precipitação química. Esta técnica foi escolhida pois, além de ser a mais estudada [22, a síntese é simples [23, 24], barata [23] e a reação ocorre a uma faixa ampla de temperatura, podendo ser realizada tanto em temperatura elevada quanto em temperatura ambiente [25], o que facilita sua execução em sala de aula.

Este método consiste na mistura estequiométrica de sais que contenham íons ferrosos $\left(\mathrm{Fe}^{2+}\right)$ e férricos $\left(\mathrm{Fe}^{3+}\right)$, os quais são precipitados na presença de um meio alcalino na forma de magnetita $\left(\mathrm{Fe}_{3} \mathrm{O}_{4}\right)[22,[25] 28]$ ou maghemita $\left(\gamma-\mathrm{Fe}_{2} \mathrm{O}_{3}\right)[25,26,29]$. De fato, esta metodologia produz NPmag geralmente polidispersas [24, 26, 30], e o tamanho, forma e composição destas nanoestruturas dependerão dos tipos de sais usados, da razão molar de $\mathrm{Fe}^{2+}$ e $\mathrm{Fe}^{3+}$, das condições de temperatura, $\mathrm{pH}$ e força iônica [22, 25, 26]. Sendo assim, as propriedades magnéticas também dependerão destas condições 28 .

Um dos principais fatores que afetam as propriedades das NPmag é o tamanho 31. A síntese de estruturas mesoporosas por agregação de NPmag, por exemplo, pode ser interessante em aplicações biomédicas, visto que fármacos ou enzimas podem ser incorporados nestas mesoestruturas para formar um suporte híbrido, favorecendo assim, alta carga de fármacos [28]. Além disso, partículas de tamanho na faixa nanométrica podem passar pelos vasos capilares para se ligar a uma proteína ou DNA, enquanto partículas micrométricas reagirão apenas com as células 31 33.

Além de aplicações biomédicas as NPmag também podem ser usadas como adsorventes para remoção de corantes dispersos em águas residuais 34, 35. Estas nanoestruturas oferecem vantagens em relação a outros adsorventes, pois podem remover compostos indesejados apenas com aplicação de um campo magnético externo. Nestes casos, pode-se controlar a morfologia incorporando outros compostos que facilitem o processo de adsorção. Além disso, pode-se monitorar o tamanho destas nanoestruturas para que não percam seu poder de magnetização [36. Por exemplo, a adição lenta de hidróxido de sódio $(\mathrm{NaOH})$ como um precipitante (em quantidades estequiométricas) durante a reação de coprecipitação promove agregação e formação de um material mesoporoso com alta saturação magnética [28, 37].

Com efeito, os estudos com NPmag são promissores cientificamente e as diferentes morfologias que estas estruturas podem adquirir ao longo de sua preparação pode levar a variadas aplicações [24, 36, 38]. Neste estudo deseja-se apresentar uma metodologia para obtenção de NPmag a partir de materiais do cotidiano, para alunos de Ensino Médio. Sendo assim a técnica foi adaptada para ser desenvolvida com estes materiais sem a preocupação de se desenvolver NPmag com características específicas.

Na realização do experimento para a obtenção das NPmag foram utilizados os seguintes materiai, ${ }^{1}$

- Vinagre de álcool;

- Palha de aço número 2;

- Solução à base de amônia;

- Água oxigenada;

- Ímã retangular de $5 \mathrm{~cm}$;

- Hidróxido de amônio $\left(\mathrm{NH}_{4} \mathrm{OH}\right)$;

- Recipiente transparente de vidro com capacidade superior a $200 \mathrm{~mL}$;

- Balança de cozinha.

\subsection{Preparação das nanopartículas magnéticas}

A primeira etapa desta proposta foi adaptar a proposta de Galiazzi 39] para desenvolver uma metodologia para preparação das NPmag a partir de materiais do cotidiano. As nanopartículas magnéticas foram preparadas a partir de soluções de $\mathrm{Fe}^{2+}$ e $\mathrm{Fe}^{3+}$.

A solução de $\mathrm{Fe}^{2+}$ foi preparada a partir da adição de $200 \mathrm{~mL}$ de vinagre em 4,0 g de palha de aço. A solução foi deixada em repouso por $20 \mathrm{~h}$. O vinagre é um condimento líquido que contém compostos bioativos, como ácidos orgânicos, principalmente ácido acético, e compostos fenólicos [40]. A concentração de ácido acético total presente no vinagre comercial no Brasil é de 4\% 41, 42. O ácido acético, presente no vinagre, aumenta a concentração de íons $\mathrm{H}^{+}$no meio, o que acelera a reação de oxidação da palha de aço. Já a palha de aço é uma liga metálica formada essencialmente por ferro -Fe- e carbono [43, sendo o ferro o elemento predominante (aproximadamente 98\%) [38, 44, 45]. O ferro na presença do oxigênio do ar sofre oxidação, enquanto o oxigênio sofre redução (agente oxidante) transformando-se em íons $\mathrm{Fe}^{2+}$ (Reação 1). A solução apresenta coloração amarelada associada à formação de $\mathrm{Fe}^{2+}[46]$.

$$
2 \mathrm{Fe}(\mathrm{s})+\mathrm{O}_{2}(\mathrm{~g})+4 \mathrm{H}^{+}(\mathrm{aq}) \rightarrow 2 \mathrm{Fe}^{2+}(\mathrm{aq})+2 \mathrm{H}_{2} \mathrm{O}(\mathrm{l})
$$

A solução de $\mathrm{Fe}^{3+}$ foi preparada adicionando-se 20 gotas de água oxigenada em $15 \mathrm{~mL}$ de solução de $\mathrm{Fe}^{2+}$ preparada anteriormente. A reação entre o $\mathrm{Fe}^{2+}$ e o peróxido de hidrogênio, presente na água oxigenada,

\footnotetext{
1 Como a marca dos materiais pode influenciar nos resultados, as marcas utilizadas neste trabalho foram: Vinagre de álcool (Chemim); Palha de aço (Veja); Solução de amônia e solução de peróxido de hidrogênio (Farmax); Hidróxido de amônio (Biotec).
} 
é conhecida como "reação tipo Fenton" sendo um dos sistemas mais ativos para a oxidação de compostos orgânicos em água [47. A reação de Fenton se baseia na decomposição de peróxido de hidrogênio a formas intermediárias de radicais livres (Reação 2), os quais possuem potencial de redução maior que o próprio peróxido 47 49], atuando como um catalisador homogêneo [47. Esta solução produzida após a reação tem coloração avermelhada, o que evidencia a presença do $\mathrm{Fe}^{3+}[50]$.

$$
\mathrm{Fe}^{2+}+\mathrm{H}_{2} \mathrm{O}_{2}(\mathrm{aq}) \rightarrow \mathrm{Fe}^{3+}+{ }^{-} \mathrm{OH}+{ }^{\bullet} \mathrm{OH} \quad(\text { Reação } 2)
$$

Outra forma de identificar os compostos $\mathrm{Fe}^{2+} \mathrm{e} \mathrm{Fe}^{3+}$ é a partir da adição nas soluções previamente preparadas de hidróxido de amônio $\left(\mathrm{NH}_{4} \mathrm{OH}\right)$ [1] (Reação 3) e (Reação 4). A solução formada com o $\mathrm{Fe}^{2+}$ apresenta coloração branca acinzentada [52]. Cabe ressaltar que o $\mathrm{Fe}(\mathrm{OH})_{2}$ é um redutor bastante forte sendo facilmente oxidado pelo ar, formando $\mathrm{Fe}(\mathrm{OH})_{3}[53$, logo este teste foi realizado apenas para identificação do composto. Já a solução contendo $\mathrm{Fe}^{3+}$ possui uma coloração marrom avermelhado [51, 53.

$$
\begin{aligned}
& \mathrm{Fe}^{2+}+2 \mathrm{NH}_{4} \mathrm{OH} \rightarrow \mathrm{Fe}(\mathrm{OH})_{2}+2 \mathrm{NH}_{4}^{+} \\
& \mathrm{Fe}^{3+}+3 \mathrm{NH}_{4} \mathrm{OH} \rightarrow \mathrm{Fe}(\mathrm{OH})_{3}+3 \mathrm{NH}_{4}^{+}
\end{aligned}
$$

Após a etapa de identificação dos íons $\mathrm{Fe}^{2+}$ e $\mathrm{Fe}^{3+}$ as NPmag foram sintetizadas por coprecipitação em meio alcalino dos íons acima. Para isso, misturou-se 25 $\mathrm{mL}$ de $\mathrm{Fe}^{2+}, 15 \mathrm{~mL}$ de $\mathrm{Fe}^{3+}, 5 \mathrm{~mL}$ de hidróxido de amônio $\left(\mathrm{NH}_{4} \mathrm{OH}\right)$, que é o agente precipitante [54, e 10 $\mathrm{mL}$ de amônia $\left(\mathrm{NH}_{3}\right)$, que atua como um catalisador básico [55]. Segue abaixo (Reação 5) um exemplo de reação de coprecipitação formada [23].

$$
\mathrm{Fe}^{2+}+2 \mathrm{Fe}^{3+}+8 \mathrm{OH} \rightarrow \mathrm{Fe}_{3} \mathrm{O}_{4}+4 \mathrm{H}_{2} \mathrm{O}
$$

(Reação 5)

A reação é rápida, de alto rendimento e os cristais de magnetita $\left(\mathrm{Fe}_{3} \mathrm{O}_{4}\right)$ se apresentam na forma de um precipitado de cor preta [56]. Além disso estas NPmags, quando expostas a um meio aeróbio, são gradualmente oxidadas à maghemita $\left(\gamma-\mathrm{Fe}_{2} \mathrm{O}_{3}\right)$ (Reação 6 [56, 57].

$$
2 \mathrm{Fe}_{3} \mathrm{O}_{4}+\frac{1}{2} \mathrm{O}_{2} \rightarrow \gamma-\mathrm{Fe}_{2} \mathrm{O}_{3}
$$

(Reação 6)

A solução final foi deixada em repouso por $1 \mathrm{~h}$. O frasco com a suspensão escura contendo as nanopartículas magnéticas (NPmag) foi transferido para um tubo de ensaio, onde, com o auxílio de um ímã, podese verificar a formação das nanopartículas (Fig. 1). As NPmag decantadas foram centrifugadas (modelo Z 36 HK1, marca Hermle) a uma rotação de 10 mil rpm por dez minutos.

\subsection{Métodos para caracterização das nanopartículas magnéticas}

Para saber se as partículas obtidas podem ser definidas como nanopartículas, antes é necessário que elas passem

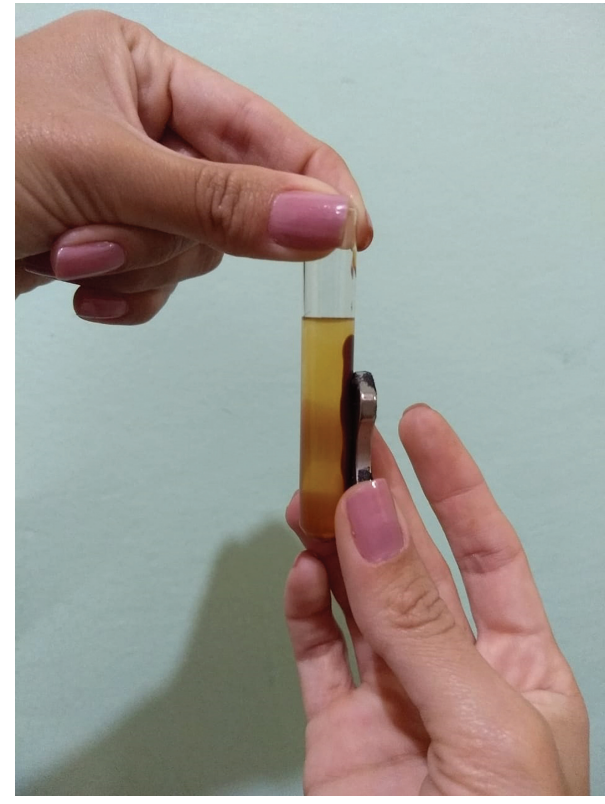

Figura 1: Solução de NPmag orientadas na presença de um campo magnético externo (na presença de um imã).

por um processo de caracterização, que também indica se o método para obtenção delas foi eficiente ou não. Dentre os métodos existentes para caracterização de materiais na escala nanométrica, neste trabalho, escolheu-se as técnicas de Espalhamento de Luz Dinâmico (Dynamic Light Scattering - DLS) e a Microscopia Eletrônica de Varredura (MEV). Essas técnicas são eficientes para determinação do tamanho e morfologia das estruturas, no entanto, elas não são acessíveis aos laboratórios didáticos das escolas de Educação Básica. Desse modo, este trabalho apresenta resultados dessa caracterização, obtidos por meio de colaboração com a Universidade Estadual do Centro - Oeste, para que os professores possam apresentar e discutir esses resultados com os estudantes.

A técnica de Espalhamento de Luz Dinâmico (DLS) foi utilizada para medir o tamanho das NPmag sintetizadas. Nesta técnica, a suspensão contendo as NPmag é exposta a um feixe de luz (onda eletromagnética) e a direção e a intensidade do feixe incidente são alterados devido a um processo de espalhamento. Como as NPmag estão em um movimento aleatório constante devido à sua energia cinética, a variação da intensidade com o tempo contém informações sobre este movimento aleatório e pode ser usado para medir o coeficiente de difusão $(D)$ 58 60. Para partículas esféricas o raio hidrodinâmico $\left(R_{h}\right)$ da partícula pode ser calculado pela equação de Stokes-Einstein (Equação (1)), onde $k_{B}$ é a constante de Boltzmann, $T$ é a temperatura da suspensão e $\eta$ é a viscosidade do meio circundante [59, 60.

$$
R_{h}=\frac{k_{B} T}{6 \pi \eta D}
$$

Equação 1. Equação de Stokes-Einstein 


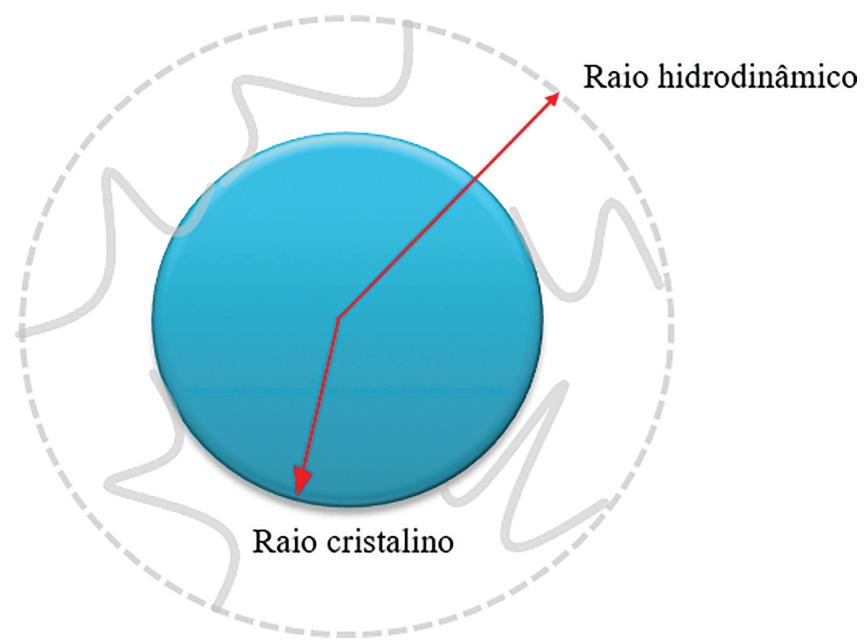

Figura 2: Esquema comparando o raio hidrodinâmico e raio cristalino de uma partícula esférica e solvatada.

Em resumo, nesta técnica, o raio hidrodinâmico é o raio de uma esfera que tem o mesmo coeficiente de difusão dentro do mesmo ambiente viscoso das partículas sendo medidas e está diretamente relacionado ao movimento difusivo destas [58, 60. Além disso, devido ao fato da metodologia utilizar luz visível como fonte de energia e ângulos facilmente obtidos para detecção da luz espalhada, pode-se dimensionar partículas em solução com tamanhos entre $2 \mathrm{~nm}$ e $500 \mathrm{~nm}$ ou sua distribuição de tamanho [59, 60].

Cabe ressaltar também, de acordo com Silveira e Giacomelli, que muitas partículas não são esféricas e estão solvatadas (Fig. 2). Sendo assim, o valor do raio hidrodinâmico reflete um tamanho aparente da partícula levando-se em conta a sua hidratação ou solvatação [59].

O equipamento utilizado na técnica de DLS foi o Zetasizer NanoZS Malvern. Para esta análise o precipitado foi recolhido com um ímã forte e o sobrenadante foi removido do precipitado por decantação. Em seguida as NPmag foram dispersas em água MiliQ na proporção NPmag/água MiliQ de 2:100 e foram novamente centrifugadas para remoção das impurezas. Após, a amostra foi carregada em uma célula de dispersão termostatizada a $25^{\circ} \mathrm{C}$ e as análises foram realizadas. As medidas foram realizadas em triplicata e foi considerada a média das distribuições de tamanho das partículas.

Inicialmente, a partir da análise de DLS, verificouse a presença de partículas de diferentes tamanhos (fisicamente diz-se que o sistema é polidisperso), o que se atribui à presença de agregados. Para comprovar se realmente o sistema é polidisperso verificou-se, durante a análise, um fator conhecido como Índice de Polidispersão (PdI). Para uma melhor qualidade da amostra, em termos de uniformidade de tamanho, os valores de PdI devem variar de 0 a 1 e quanto mais próximo ao zero melhor será 60. Além disso sabe-se que valores de PdI $<0,1$ indicam a presença de amostras monodispersas 60 , 61] e consequentemente, quanto mais afastado deste

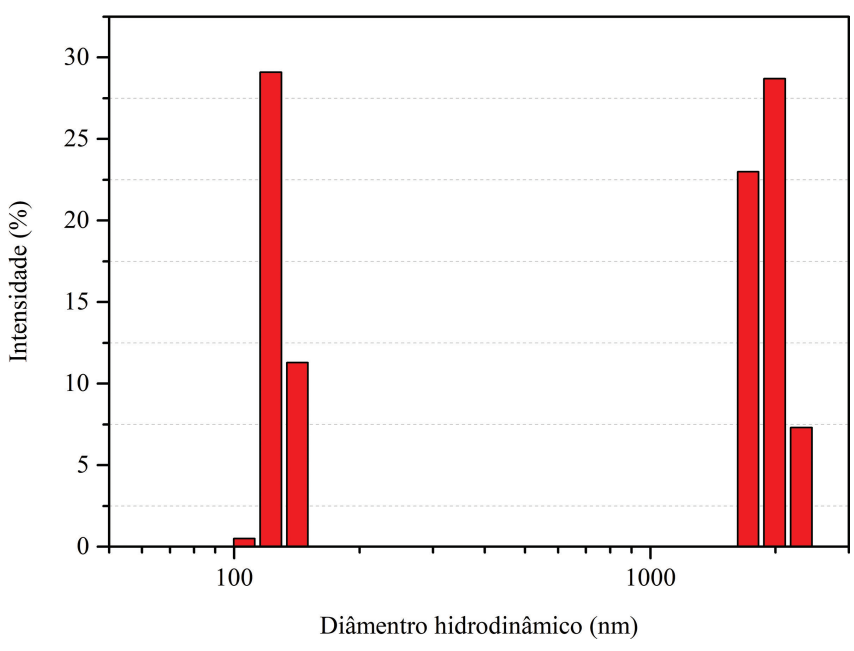

Figura 3: Histograma para análise do diâmetro hidrodinâmico médio das NPmag desenvolvidas a partir da técnica de DLS.

valor mais polidispersa será a amostra. Neste trabalho os valores encontrados para a polidispersão (PdI) das NPmag produzidas foram altos (em torno de 0,434 ) o que confirma que o sistema produzido foi polidisperso. Essa polidispersão já era esperada visto que a metodologia escolhida já previa que o sistema formado teria esta característica [62].

Como o sistema é polidisperso a melhor forma de entender qual a distribuição de tamanho presente é através de um histograma (Fig. 3). O histograma apresenta a média de diâmetros hidrodinâmicos obtidos através da intensidade da luz incidente. Conforme verifica-se no histograma os valores médios dos diâmetros hidrodinâmicos das NPmag desenvolvidas ficaram entre 100 e $2400 \mathrm{~nm}$.

Como estes valores são altos acredita-se que estes diâmetros representam na realidade o tamanho dos agregados gerados pelas NPmag. De acordo com a literatura 63] a tendência de agregação é uma propriedade física crucial em NPmag. Este tipo de estrutura possui forças de van der Waals e interações dipolo-dipolo magnéticas geradas a partir de momentos magnéticos residuais, que tendem a aglomerar as partículas 64. Ou seja, neste tipo de suspensão os encontros entre as partículas dispersas em meios líquidos ocorrem com frequência, e a estabilidade da suspensão é determinada pela interação entre as partículas durante esses encontros. Cabe ressaltar que uma solução coloidal é uma suspensão em que a fase dispersa é tão pequena que as forças gravitacionais são desprezíveis e as interações das partículas são dominadas por forças de curto alcance, tal como as forças de atração de van der Waals [63. e as forças de longo alcance [65, tais como as cargas superficiais. A principal causa da agregação então serão a presença destas forças 63 .

Logo, para promover uma dispersão estável, forças repulsivas de curto alcance são necessárias para manter cada partícula discreta e evitar que ela se acumule em aglomerados maiores e de sedimentação mais rápida. 
O impedimento estérico é uma das principais forças de superfície e desempenham um papel importante na estabilização das suspensões. Isso pode ser realizado ligando estericamente moléculas orgânicas de cadeia longa à superfície do óxido que atuarão como escudos protetores [63, 64, 66. A força repulsiva resulta na criação de uma dupla camada elétrica ao redor das partículas, que pode evitar a formação de agregados [63, 64].

Por outro lado, como dito acima, NPmag dispersas em solução também são compostas por momentos magnéticos não-nulos [25, 67]. A interação dipolar magnética entre uma partícula e suas vizinhas dependerá da orientação relativa dos momentos magnéticos e do produto de suas intensidades. Desta forma, interações com um caráter atrativo por exemplo, podem induzir a formação de agregados ao sistema o que pode dar uma ideia equivocada quanto ao diâmetro. Ademais, como informado ao longo deste texto, a técnica DLS está associada a estruturas envolvidas em suas camadas de solvatação, o que também pode superestimar o tamanho médio determinado [59,60] . O resultado obtido para esta análise está de acordo com os estudos encontrados na literatura sobre o assunto 68, 69.

A técnica de DLS sozinha é incapaz de distinguir a forma das partículas. Sendo assim, análises de MEV foram realizadas, já que esta técnica, além de fornecer uma ideia quanto a morfologia, também pode oferecer uma estimativa do diâmetro da NPmag.

As imagens de MEV foram obtidas utilizando um microscópio de feixe de íon focalizado FEI Scios operando com um feixe de elétrons na tensão de $20 \mathrm{kV}$. Para a preparação das amostras, as nanopartículas produzidas foram dispersas em um béquer com acetona e agitado em banho ultrassônico por $5 \mathrm{~min}$. Em seguida, uma gota da dispersão foi depositada em um suporte metálico, e uma fina camada de ouro foi depositada a fim de promover a condutividade superficial da amostra. O ouro foi depositado pelo processo de sputtering em um metalizador Shimadzu IC-50.

A Figura 4a e 4p apresentam as imagens de MEV das NPmag produzidas. A análise dos resultados foi feita através de inspeção visual. A primeira imagem analisada (Fig. 4a) foi observada com uma ampliação de 50 mil vezes, visualizada em uma escala de $1 \mu \mathrm{m}$. A partir dela observa-se que o sistema estudado é constituído por agregados magnéticos cuja distribuição de tamanho é larga, na ordem de $2000 \mathrm{~nm}$ (ou 2,0 $\mu \mathrm{m}$ ). Acredita-se, conforme teoria apresentada anteriormente, que estes agregados se devem a atração magnética destas estruturas. Tal evidência confirma os resultados identificados na análise de DLS e são confirmados em outros estudos sobre o assunto [28, 36], elucidando a discussão.

Além de ser possível observar os tamanhos das amostras produzidas, a partir da técnica de MEV também é possível verificar a morfologia destas estruturas. A técnica de MEV é uma das técnicas mais versáteis disponíveis para isso 21. Na obtenção das imagens para o estudo da morfologia são mostradas ampliações de 240 mil vezes, visualizadas em uma escala de $300 \mathrm{~nm}$ (Fig. 4 p).

Verifica-se que os agregados são esféricos e são formados por estruturas de formato irregular com uma tendência esférica. De acordo com a literatura esta morfologia é característica da presença das fases magnetita/maghemita [23, 31, 70,72]. Além disso, este resultado também é condizente com os obtidos em outros estudos 69, 68.

Com o objetivo de aprofundar a análise das imagens, construiu-se um histograma relativo à distribuição de tamanho médio das NPmag. Para isso calculou-se a média o diâmetro de cerca de 100 partículas presentes na imagem $4 \mathrm{~b}$. Em seguida construiu-se o histograma do número de partículas em função do tamanho médio das partículas (com ajuste matemático usando uma distribuição Log-normal) (Fig. 5.

Analisando o histograma constatou-se uma distribuição de tamanho larga com diâmetros variando de $18 \mathrm{~nm}$ até aproximadamente $65 \mathrm{~nm}$, observando uma maior frequência em torno de $35 \mathrm{~nm}$ a $40 \mathrm{~nm}$.

Uma das hipóteses para a predominância destes tamanhos pode estar relacionada às condições de preparo das

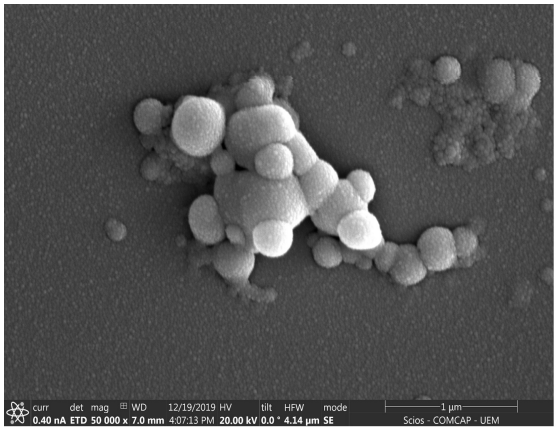

(a)

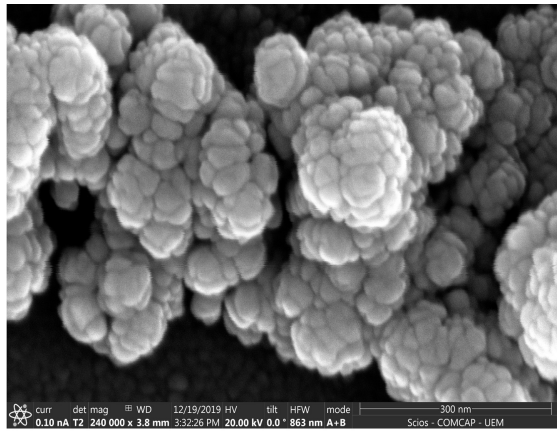

(b)

Figura 4: a. Imagem de MEV das NPmg desenvolvidas com uma ampliação de 50 mil vezes. b. Imagem de MEV das NPmg desenvolvidas com uma ampliação de 240 mil vezes. 


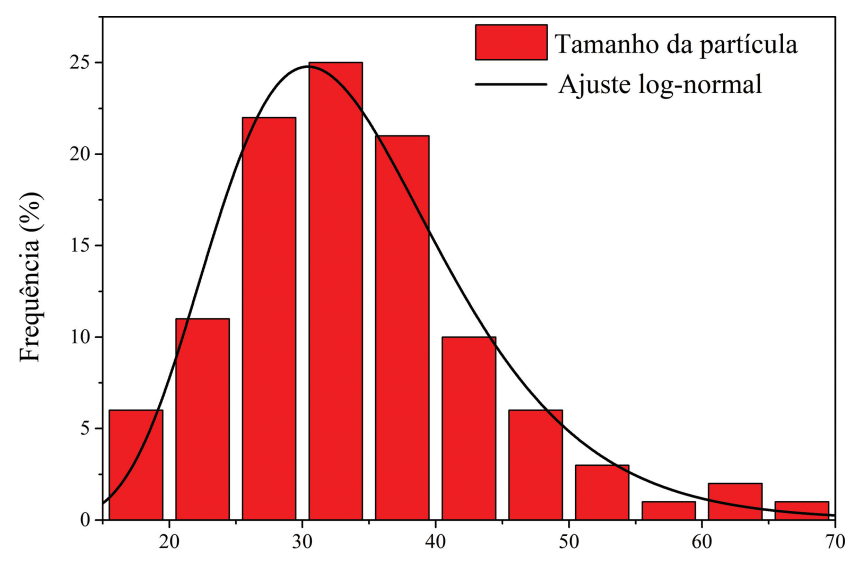

Distribuição de tamanho das partículas (nm)

Figura 5: Histograma para análise do diâmetro hidrodinâmico médio das NPmag produzidas a partir da técnica de MEV. Linha ajuste: distribuição Log-normal.

nanopartículas, tais como temperatura, $\mathrm{pH}$ e escolha do agente precipitante na metodologia. $\mathrm{O}$ agente escolhido foi o $\mathrm{NH}_{4} \mathrm{OH}$, que, de acordo com a literatura [62], consegue formar nanoestruturas com maior estabilidade e mais controláveis do que se o $\mathrm{NaOH}$, uma base mais forte [73. O álcali adequado pode influenciar, tanto o diâmetro das NPmag, como na resposta magnética. Em $\mathrm{NH}_{4} \mathrm{OH}$ a concentração de $\mathrm{OH}^{-}$é liberada gradualmente o que controla o que controla com mais facilidade o tamanho da partícula [62. Os resultados obtidos nesta etapa do trabalho indicam que a metodologia é eficiente e forma NPmag, podendo, portanto, ser aplicada em sala de aula.

\section{Proposta Didática para Estudo de Nanotecnologia e Nanociência no Ensino Médio}

A técnica de obtenção de NPmag, descrita acima, foi implementada em uma abordagem CTSA para o ensino de Nanociência e Nanotecnologia. A proposta didática, além de promover uma interrelação entre ciência, tecnologia, sociedade e ambiente, também foi planejada levando em consideração aspectos de teorias de aprendizagem construtivistas, que valorizam o conhecimento prévio dos alunos e a proposta de materiais potencialmente significativos e relacionados ao contexto dos estudantes [74, 75].

Essa abordagem didática foi realizada com alunos do primeiro ano do Ensino Médio, após encerrar o conteúdo relacionado ao Sistema Internacional de Medidas (SI) e começar a trabalhar com notação científica, momentos no qual foi apresentada a tabela de prefixos do Sistema Internacional de Unidades (SI). Nesta etapa se discutiu a relevância desses prefixos para descrever medidas de diversas grandezas físicas, indo desde a distância entre corpos celestes até dimensões de partículas invisíveis a olho nu. No quadro 1 é apresentada a estrutura da abordagem didática utilizada.

Aula 01: A primeira aula foi destinada a obtenção dos conhecimentos prévios dos estudantes a respeito da temática e também para a explanação inicial do conteúdo. Para isso, foi utilizado um questionário inicial, composto pelos seguintes questionamentos:

1) De acordo com os seus conhecimentos o que é nanociência?

2) De acordo com os seus conhecimentos o que é nanotecnologia?

3) Em seu cotidiano você utiliza de nanotecnologia?

4) Cite exemplos de aplicações da nanociência.

Após os alunos responderem ao questionário a professora deu início à explanação do conteúdo utilizando uma apresentação de slides a respeito do tema. Nesta apresentação foram exibidas definições da temática e áreas nas quais a nanociência é relevante, bem como a presença da mesma em materiais e produtos que são utilizados no cotidiano. Para concluir a primeira aula, foi apresentada aos alunos uma animação, em forma de vídeo, que resumia aspectos importantes da explanação do conteúdo, como um meio de representar o que havia sido exposto de uma forma diversificada.

Aula 02: Na segunda aula os alunos foram orientados para o desenvolvimento de uma atividade prática relacionada ao tema. A atividade escolhida foi a obtenção de nanopartículas magnéticas com material de baixo custo, descrita no item 2 deste artigo.

Primeiramente os alunos pesaram a palha de aço e em seguida a colocaram em um recipiente de vidro acrescentando $200 \mathrm{~mL}$ de vinagre de álcool. O próximo passo foi deixar essa amostra em repouso por um período de 20 h. Para adiantar o processo, e não ser necessário esperar este tempo, a professora preparou as amostras no dia anterior o que permitiu dar andamento a atividade. No entanto, explicou-se aos alunos a necessidade de deixar as amostras em repouso pelo período indicado para que ocorresse o processo de oxirredução, para a formação do $\mathrm{Fe}^{+2}$.

Em seguida os alunos fizeram todos os procedimentos para obtenção das reações 2, 3, 4 e 5 descritas no tópico 2 deste artigo. Para isso os alunos receberam um roteiro no qual estavam descritos os materiais que seriam utilizados e o passo a passo de como realizar o experimento. Ao término da aula as amostras foram guardadas para serem observadas na próxima aula.

Aula 03: Na terceira aula as amostras foram levadas para sala de aula e os alunos puderam observar as partículas que se formaram. Para tal, utilizaram um ímã nas paredes externas dos recipientes que continham as amostras. Foram utilizados dois recipientes diferentes, um tubo de ensaio e um vidro de relógio.

Em seguida a professora explicou como era feito o processo de medida das dimensões dessas partículas, um procedimento que precisa de equipamentos que não 
Quadro 1: Estrutura da Abordagem Didática.

\begin{tabular}{|c|c|}
\hline \multicolumn{2}{|r|}{ Aula 01} \\
\hline Atividades & Descrição \\
\hline $\begin{array}{l}\text { Obtenção dos } \\
\text { conhecimentos prévios } \\
\text { dos estudantes e } \\
\text { questionamentos } \\
\text { iniciais. }\end{array}$ & $\begin{array}{l}\text { No início da aula os alunos responderam um questionário referente ao tema proposto, } \\
\text { com objetivo de obter os conhecimentos prévios dos estudantes a respeito do tema e ao } \\
\text { mesmo tempo provocar a curiosidade dos alunos em relação à temática proposta. }\end{array}$ \\
\hline $\begin{array}{l}\text { Explanação do } \\
\text { Conteúdo }\end{array}$ & $\begin{array}{l}\text { A explanação do conteúdo foi desenvolvida utilizando como recurso didático uma } \\
\text { apresentação em slides para trabalhar com o tema nanociência, nanotecnologia e suas } \\
\text { aplicações. }\end{array}$ \\
\hline $\begin{array}{l}\text { Animação: O que é } \\
\text { nanotecnologia? }\end{array}$ & $\begin{array}{l}\text { Apresentação da animação: O que é Nanotecnologia }{ }^{2} \text { ? } \\
\text { Essa atividade teve como objetivo apresentar de forma dinâmica e contextualizada o } \\
\text { conceito de nanoescala e resumir o conteúdo apresentado para a conclusão da primeira } \\
\text { aula. }\end{array}$ \\
\hline \multicolumn{2}{|r|}{ Aula 2} \\
\hline Atividade Experimental & $\begin{array}{l}\text { A segunda aula foi destinada para a realização de uma atividade prática relacionada ao } \\
\text { tema discutido na aula anterior. Durante esta aula os alunos foram orientados e } \\
\text { desenvolveram a prática experimental de obtenção de nanopartículas magnéticas com } \\
\text { material de baixo custo, descrita acima. }\end{array}$ \\
\hline \multicolumn{2}{|r|}{ Aula 3} \\
\hline $\begin{array}{l}\text { Discussão do resultado } \\
\text { experimental e } \\
\text { contextualização do } \\
\text { conteúdo. }\end{array}$ & $\begin{array}{l}\text { Na terceira aula, foram discutidos os resultados da prática experimental e em seguida } \\
\text { foram apresentadas relações entre o conteúdo abordado e aplicações da nanociência e } \\
\text { nanotecnologia na solução de problemas ambientais, tecnológicos e de saúde pública. }\end{array}$ \\
\hline Atividade Final & $\begin{array}{l}\text { Para fins de avaliação do processo de ensino e aprendizagem os alunos responderam ao } \\
\text { questionário posterior, composto pelas mesmas questões do questionário inicial. }\end{array}$ \\
\hline
\end{tabular}

estão disponíveis nos laboratórios didáticos das escolas estaduais e que devido a isso, estava sendo apresentado para eles os resultados de um procedimento de medida realizado em uma universidade.

A discussão dos resultados da prática experimental foi finalizada com uma apresentação a respeito do uso da nanotecnologia na sociedade atual e suas potencialidades na solução de problemas ora ambientais, tecnológicos ou voltados para questões de saúde pública. Além disso, foram citados alguns materiais e produtos, que são aplicações de nanociência, para que eles fizessem a relação do conteúdo estudado com seu cotidiano.

A proposta didática foi realizada durante três aulas em uma turma de primeiro ano do Ensino Médio, de uma Escola Estadual localizada na cidade de Turvo, região centro-oeste do Estado do Paraná. A turma foi composta por 8 alunos com faixa etária de 14 a 16 anos.

\section{Resultados da Abordagem Didática}

Os dados aqui apresentados e discutidos foram obtidos por meio de questionários e anotações da professora da turma. O procedimento de análise foi realizado seguindo

2 Disponível em: https://www.youtube.com/watch?v=oHgN4jtie Tg\&feature=youtube acesso em 27/04/2020. pressupostos da Análise de Conteúdo [76]. Foram elaboradas Unidades de Contexto (UC) e de Registro (UR) para cada uma das questões propostas e as respostas dos alunos foram classificadas entre as unidades de registro (UR). Os dados aqui utilizados são oriundos das respostas formalmente esclarecidas e consentidas.

Para manter preservada a identidade dos estudantes, refere-se aos mesmos com códigos: A1, A2, A3, A4, A5 e A6. Para a análise dos questionários não são utilizados os dados de dois alunos, A7 e A8, devido terem faltado na terceira aula.

A seguir, no quadro 2 são apresentadas as noções dos estudantes a respeito dos conceitos de nanociência e nanotecnologia.

Como pode-se perceber no quadro 2, o conhecimento inicial dos estudantes a respeito do tema nanociência e nanotecnologia era inexistente. Todos os alunos afirmam não conhecer ou não recordar nada em relação a esses temas. Esses resultados corroboram com resultados iniciais de outras pesquisas como o estudo desenvolvido por Oliveira, Ferreira e Almeida [77]. Esse mapeamento inicial dos conhecimentos dos estudantes é essencial para que se identifique o nível e as falhas de conhecimento, a fim de que os professores possam atuar diretamente sobre os problemas identificados.

Em relação ao questionário posterior, em ambas as questões, os alunos passaram a apresentar alguma noção 
Quadro 2: Dados referentes às questões 01 e 02.

UC1 "Noções a respeito de nanociência": tem por finalidade reunir os fragmentos textuais nos quais os alunos expressam suas noções a respeito do que entendem por nanociência.

Unidades de Registro $\quad$ Registros

\begin{tabular}{lll}
\hline UR & PRÉVIO & POSTERIOR
\end{tabular}

UR 1.1 "Noções de acordo com o consenso científico atual"

UR 1.2 "Noção parcialmente correta a respeito do tema"

UR 1.3 "Noções equivocadas/confusas em relação ao tema"

UR 1.4 "Desconhece ou não recorda nada em relação ao tema"
"Não sei" (A1)

"É o ramo que estuda materiais, propriedades $e$ comportamentos de escala nanométrica. Podemos encontrar elas na medicina, na química, na biologia, na informática, na física e na engenharia." (A1)

Número de registros: 02 (A1 e A3)

"É a tecnologia que usamos hoje em dia como celulares, notebook, entre outros." (A6)

Número de registros: 03 (A4, A5 e A6)

"Nanociência, ramo que estuda a matéria $e$ propriedade e comportamento da matéria." (A2)

Número de registros: 01 (A2)

(A1, A2, A3, A4, A5 e A6)

UC2 "Noções a respeito de nanotecnologia", que tem por finalidade reunir os fragmentos textuais nos quais os alunos expressam suas noções a respeito do que entendem por nanotecnologia.

\begin{tabular}{|c|c|c|}
\hline Unidades de Registro & & úmero de Registros \\
\hline$\overline{\mathbf{U R}}$ & PRÉVIO & POSTERIOR \\
\hline $\begin{array}{l}\text { UR } 2.1 \text { "Noções de acordo } \\
\text { com o consenso científico }\end{array}$ & & $\begin{array}{l}\text { "É aquela que manipula átomos e moléculas na } \\
\text { nanoescala." (A5) }\end{array}$ \\
\hline atual" & & Número de registros: $04(\mathrm{~A} 2, \mathrm{~A} 4, \mathrm{~A} 5$ e $\mathrm{A} 6)$ \\
\hline $\begin{array}{l}\text { UR } 2.2 \text { "Noção } \\
\text { parcialmente correta a } \\
\text { respeito do tema" }\end{array}$ & & \\
\hline $\begin{array}{l}\text { UR } 2.3 \text { "Noções } \\
\text { equivocadas/confusas em } \\
\text { relação ao tema" }\end{array}$ & & $\begin{array}{l}\text { "O nanômetro corresponde a bilionésima parte } \\
\text { do metro, ou seja, a menor coisa que podemos } \\
\text { ver a olho nu é o fio de cabelo e a escala } \\
\text { manométrica é menor que isso." (A1) }\end{array}$ \\
\hline
\end{tabular}

UR 2.4 "Desconhece ou não recorda nada em relação ao tema"
"Não sei" (A1)

Número de registros: 02 (A1 e A3)
Número de registros: 06 (A1, A2, A3, A4, A5 e A6) em relação aos termos estudados. Dentre as respostas, duas podem ser classificadas como coerentes com o consenso científico atual a respeito de nanociência, nas quais o termo é descrito como o estudo de estruturas na escala nanométrica [2] e quatro podem ser classificadas como coerentes em relação ao termo nanotecnologia, nas quais o termo é descrito como a manipulação e aplicação dessas estruturas nanométricas [2].

Os demais alunos apresentaram noções parcialmente corretas (03 registros) ou equivocadas (01 registro) em relação a nanociência e noções confusas (02 registros) em relação a nanotecnologia.
A seguir, no quadro 3 são apresentados os dados referentes às noções dos estudantes a respeito da aplicação de nanociência e nanotecnologia.

Com base nas UR 3.4 e UR 4.4, em relação aos conhecimentos prévios, percebe-se que eles não sabem opinar a respeito das possíveis aplicações de nanociência e nanotecnologia, o que é de se esperar. Além disso, eles também não sabiam definir esses conceitos nas questões 01 e 02 , do questionário prévio.

No entanto, após a abordagem didática dos temas, os alunos passaram a apresentar noções de conhecimento de possíveis aplicações da nanociência e nanotecnologia, 
Quadro 3: Dados referentes às questões 03 e 04.

UC3 "Noções a respeito do uso de nanotecnologia no cotidiano", que tem por finalidade reunir os fragmentos textuais nos quais os alunos expressam suas noções a respeito da utilização de nanotecnologia no cotidiano.

\begin{tabular}{lcc}
\hline Unidades de Registro & Número de Registros \\
\hline UR & PRÉVIO & POSTERIOR \\
\hline UR 3 "Não utiliza" &
\end{tabular}

UR 3.1 "Não utiliza"

UR 3.2 "Utiliza e cita exemplos"

UR 3.3 "Noções

equivocadas/confusas em relação ao tema"

UR 3.4 "Desconhece ou não recorda nada em relação ao tema"
"Sim. Por que a gente usa a tecnologia e os cosméticos." (A3)

Número de registros: 05

(A1, A3, A4, A5 e A6)

UC4 "Noções a respeito de aplicações da nanociência", que tem por finalidade reunir os fragmentos textuais nos quais os alunos expressam suas noções a respeito das aplicações de nanociência.

Unidades de Registro Número de Registros

UR

PRÉVIO

UR 4.1 "Cita exemplos

relacionados ao meio

ambiente"

UR 4.2 "Cita exemplos

relacionados a medicina"

UR 4.3 "Noções

equivocadas/confusas em

relação ao tema"

UR 4.4 "Desconhece ou não recorda nada em relação ao tema"
"Não sei" (A1)

Número de registros: 06

(A1, A2, A3, A4, A5 e A6)
Número de Registros

"Servem para a descontaminação da água de rios poluídos, seu tamanho é na ordem de nanômetros, eles interagem com o produto e assim descontaminando a água." (A1)

Número de registros: 06 (A1, A2, A3, A4, A5 e A6)

"Para tratamento do câncer, [...], para tratamentos de doenças." (A6)

Número de registros: $\mathbf{0 3}$ (A4, A5 e A6) tanto relacionadas ao cotidiano quanto a ações mais específicas. Dentre as possíveis aplicações da nanotecnologia no cotidiano, a maioria dos alunos relacionou ao uso de celulares, computadores, cosméticos, como representado pela resposta de A3, na UR3.1.

Em relação à aplicação de nanociência, os alunos citaram exemplos de aplicações em ações voltadas ao meio ambiente e em relação a tratamento de doenças, como pode se perceber na resposta de A1 na UR 4.1 e de A2 "Para a descontaminação de rios poluídos, com tamanho na ordem de nanômetros, elas interagem com o produto que está contaminando." (A2).

Em relação à motivação dos alunos, no início eles demostraram espanto com o conteúdo que seria abordado, pois não tinham conhecimento a respeito do assunto, ou pelo menos pensavam que não. Esse desconhecimento despertou a curiosidade para que começassem estudar o tema e à medida que a professora explanava o conteúdo eles foram percebendo que já tinham lido ou escutado algo a respeito de nanociência e nanotecnologia. Isso permitiu que os discentes fossem participando da aula e fizessem comentários tais como: "professora na embalagem do esmalte está escrito a palavra "nanotecnologia", "já tinha escutado na propaganda da televisão", até a palavra "nanochip" apareceu durante a aula. Nas palavras de um dos alunos, a aula estava "fazendo sentido".

Fazer com que algum conteúdo "faça sentido" para os estudantes é um dos objetivos de abordagens como CTSA, uma vez que se espera que os conteúdos que estão 
sendo ensinados em sala de aula façam sentido aos alunos no presente e que eles percebam a relevância de estudar aquele assunto. Essa relevância fica mais evidente ao se apresentar as aplicações do conteúdo que, no caso da proposta apresentada, se deu nos momentos em que a professora apresentou exemplos de nanotecnologias aplicadas em setores ambientais e de saúde.

Além disso, o estudo desse tema passou a fazer sentido e se tornou interessante, também, pelo motivo de que os alunos foram desafiados com um problema prático. Seria possível obter nanopartículas com materiais do cotidiano? Esse desafio gerou motivação e contribuiu para que os alunos dessem significado aos conteúdos que estavam estudando.

Cabe ressaltar que a atividade que mais entusiasmou os alunos foi a prática experimental, pois ficaram impressionados com a possibilidade de eles próprios conseguirem obter NPmag em sala de aula. Na fala de um dos estudantes ouviu-se "como vamos fazer uma coisa tão pequena professora, aqui no laboratório da escola?". Quando terminou a aula teórica os alunos estavam ansiosos para a aula experimental e incrédulos que com materiais de seu cotidiano seriam capazes de obter as nanoestruturas. Durante a aula experimental eles se mostraram curiosos e cuidadosos e queriam realizar os procedimentos com calma para que o resultado não fosse prejudicado por erros procedimentais. Diante do resultado ficaram satisfeitos e animados por obter as nanopartículas e ao mesmo tempo por participar de uma aula prática.

Esse entusiasmo é um dos resultados esperados de uma abordagem CTSA, uma vez que os alunos colocam em prática conhecimentos adquiridos em sala de aula e refletem a respeito da relação entre ciência, tecnologia, sociedade e ambiente, dando sentido e significado a aprendizagem de conteúdos científicos.

\section{Conclusões}

Neste trabalho relatou-se uma proposta didática baseada em uma abordagem CTSA para produção de NPmag a partir de materiais do cotidiano. A partir da proposta foi possível obter algumas conclusões relevantes: primeiramente o método escolhido mostrou-se eficiente para a obtenção da NPmag e para aplicação em atividades de ensino. Além disso a abordagem foi capaz de contribuir para a aprendizagem de conteúdos relacionados à temática proposta, além de promover conhecimento a respeito de aplicações tecnológicas em setores ambientais e de saúde, permitindo que os discentes dessem significado ao que estavam estudando em sala de aula. Desta forma, percebe-se que a atividade possibilitou reflexões a respeito da interrelação entre ciência, tecnologia, sociedade e ambiente envolvida na compreensão do tema Nanociência e Nanotecnologia e deu significado ao seu estudo.
Ademais, o desenvolvimento da proposta didática despertou o interesse e o protagonismo dos estudantes, de modo que se espera que os resultados alcançados neste trabalho possam inspirar professores e que esta proposta se torne uma alternativa para disseminação de conhecimento científico, contextualização no Ensino de Física, promova aulas desafiadoras e proporcione o protagonismo dos discente no processo de aprendizagem, com o objetivo de alcançar uma Educação Científica contemporânea e de boa qualidade.

\section{Referências}

[1] M.L. Tomkelski, G. Scremin e S.B. Fagan, Ciência Educ. 25, 665 (2019).

[2] M.D. Tonet e A.A. Leonel, Cad. Bras. Ensino Física 36, 431 (2019).

[3] G. Sbalqueiro, L. Balvedi, R. Bettiato e J. Ribas, Rev. Saúde e Desenvolv. 12, 242 (2018).

[4] D.L. Castro, M.P. Cavalcante e M.C.G. Pedrosa, Rev. Thema. 16, 313 (2019).

[5] T.R.T. Santos, G.A.P. Mateus, M.F. Silva e R. Bergamasco, Rev. Uningá Rev. 34, 51 (2019).

[6] F.J.A. Moura, P.J.A. Silva, E.F.S. Silva, F.C.H.M. Silva, E.J.M.V.C. Feijo, Int. J. Educ. Teach. 2, 236 (2019).

[7] A.C. Fernandes, Rev. Eixo 4, 69 (2015).

[8] G.A.F. Rebello, M.M. Argyros, W.L.L. Leite, M.M. Santos, J.C. Barros, P.M.L. Santos e J.F.M. Silva, Química Nov. Na Esc. 34, 3 (2012).

[9] R.S. Abe, P.C.F. Costa e L.B. Lucas, Rev. Ensino, Educ. e Ciências Humanas 20, 260 (2019).

[10] R. Siqueira-Batista, L. Maria-Da-Silva, R.R.M. Souza, H.J. Pires-Do-Prado, C.A. Silva, G. Rôças, A.L. Oliveira e J.A. Helayël-Neto, Ciência Educ. 16, 479 (2010).

[11] W.A. Bazzo, Ciência, tecnologia e sociedade: e o contexto da educação tecnológica (Editora da UFSC, Florianópolis, 1998), v. 1, p. 34.

[12] N.A.M. Pinheiro, R.M.C.F. Silveira e W.A. Bazzo, Ciência Educ. 13, 71 (2007).

[13] W.L.P. dos Santos, E.F. Mortimer, Ens. Pesqui. Em Educ. Em Ciências. 2, 1 (2000).

[14] D. Auler, Ens. Pesqui. Em Educ. Em Ciências. 5, 1 (2003).

[15] MINISTÉRIO DA EDUCAÇÃO, Base Nacional Comum Curricular. Ensino Médio. Disponível em: http://base nacionalcomum.mec.gov.br/images/BNCC_EI_EF_11051 8_versaofinal_site.pdf

[16] MINISTÉRIO DA EDUCAÇÃO, P.C.N+ Ensino Médio Orientações Educacionais Complementares aos Parâmetros Curriculares Nacionais: Ciências da Natureza, Matemática e suas Tecnologias. Disponível em: http://portal.mec.gov.br/seb/arquivos/pdf/ciencian.pdf

[17] BRASIL, Lei no 9.394, 20 de dezembro 1996. Brasília, 1996. Disponível em: http//www.Planalto.Gov.Br/Cci vil_03/Leis/L9394.htm

[18] J. Ziman, em: STS Education: International Perspectives on Reform, editado por J. Solomon, G. Ainkenhead (Teachers College, New York, 1994a).

[19] P.N. Lisboa-Filho e M.A. Monteiro, Momento 46E, 126 (2013). 
[20] D.T. Ribeiro e P.S. Carvalho, Interacções 39, 742 (2015).

[21] E. Francisquine, J. Schoenmaker e J.A. Souza, Química Supramol. e Nanotecnologia 14, 269 (2014).

[22] R. Valenzuela, M.C. Fuentes, C. Parra, J. Baeza, N. Duran, S.K. Sharma, M. Knobel, J. Freer e J. Alloys Compd. 488, 227 (2009).

[23] S. Wu, A. Sun, F. Zhai, J. Wang, W. Xu, Q. Zhang e A.A. Volinsky, Mater. Lett. 65, 1882 (2011).

[24] J. Gallo, N.J. Long e E.O. Aboagye, Chem. Soc. Rev. 42, 7816 (2013).

[25] A.H. Lu, E.L. Salabas e F. Schüth, Angew. Chemie Int. Ed. 46, 1222 (2007).

[26] R.A. Frimpong e J.Z. Hilt, Nanomedicine 5, 1401 (2010).

[27] C. Tao, Y. Chen, D. Wang, Y. Cai, Q. Zheng, L. An, J. Lin, Q. Tian e S. Yang, Nanomaterials 9, 699 (2019).

[28] M.C. Mascolo e Y. Pei, T.A. Ring, Materials (Basel) 6, 5549 (2013).

[29] V.L. Lassalle, R.D. Zysler, M.L. Ferreira, Mater. Chem. Phys. 130, 624 (2011).

[30] C.C. Lin, J-M. Ho, Ceram. Int. 40, 10275 (2014).

[31] K. Petcharoen e A. Sirivat, Mater. Sci. Eng. B. 177, 421 (2012).

[32] H. Iida, K. Takayanagi, T. Nakanishi e T. Osaka, J. Colloid Interface Sci. 314, 274 (2007).

[33] M. Arruebo, R. Fernández-Pacheco e M.R. Ibarra, J. Santamaría, Nano Today. 2, 22 (2007).

[34] K.B. Tan, M. Vakili, B.A. Horri, P.E. Poh, A.Z. Abdullah e B. Salamatinia, Sep. Purif. Technol. 150, 229 (2015).

[35] A.F.C. Campos, P.H. Michels-Brito, F.G. Silva, R.C. Gomes e G. Gomide, J. Depeyrot, J. Environ. Chem. Eng. 7, 103031 (2019).

[36] K. Chamchoy, T. Inprasit, C. Vanichvattanadecha, A. Thiangtrong, P. Anukunwithaya e P. Pisitsak, J. Polym. Environ. 28, 1 (2020).

[37] E.T. Talgatov, A.S. Auyezkhanova, K.S. Seitkalieva, N.Z. Tumabayev, S.N. Akhmetova, A.K. Zharmagambetova, J. Porous Mater. 27, 1 (2020).

[38] L. Cornejo, H. Lienqueo, M. Arenas, J. Acarapi, D. Contreras, J. Yanez e H.D. Mansilla, Environ. Pollut. 156, 827 (2008).

[39] M.C. Galiazzi, F.P. Gonçalves, B.H. Seyffert, E.L. Hennig e J.C. Hernandes, Química Nova na Escola 21, 25 (2005).

[40] J.H. Yun, J.H. Kim, J.E. Lee, Mycobiology 47, 250 (2019).

[41] BRASIL. Instrução Normativa $\mathrm{n}^{\text {o }} 36,14$ de outubro de 1999, Disponível em: http://www.lex.com.br/doc_1655 4_INSTRUÇÃO_NORMATIVA_N_36_DE_14_DE_OUTU BRO_DE_1999.aspx.

[42] K.T. Magalhães-Guedes, R.O. Viana, R.A. Braga Jr, D.R. Dias, I.L. Nunes e R.F. Schwan, em: Ciência e Tecnologia dos Alimentos, editado por D.F. Andrade (Poisson, Belo Horizonte, 2019).

[43] S.G. Ávila e J.R. Matos, Educ. Química. 28, 254 (2017).

[44] A. López, D. Valero, L. García-Cruz, A. Sáez, V. GarcíaGarcía, E. Expósito e V. Montiel, J. Electroanal. Chem. 793, 93 (2017).

[45] Y.C. Chen, W. Liao e H.K. Yak, Ind. Eng. Chem. Res. $\mathbf{5 1 ,} 6625$ (2012).
[46] L. Wilhemy, em: O ensin exp. em Ciências Naturais, editado por Luiz Adolfo de Mello (Universidade Federal de Sergipe, São Cristóvão, 2011).

[47] J.M. Britto e M.C. Rangel, Quim. Nova 31, 114 (2008).

[48] L.C.A. Oliveira, J.D. Fabris e M.C. Pereira, Quim. Nova 36, 123 (2013).

[49] A. Aguiar, A. Ferraz, D. Contreras e J. Rodríguez, Quim. Nova 30, 623 (2007).

[50] M.E.F. Barcelos, J.R. Riguete, L.T.P. Silva, P.F. Ferreira Junior, Natureza 10, 71 (2012).

[51] L.H. Seron, Reações químicas, disponível em: http:// livresaber.sead.ufscar.br:8080/jspui/handle/123456789/ 1913 acessado em 02/06/2020.

[52] N. Pereira, M. Rocha, M. Rosa e F. Costa, Conserv. Patrimônio 34, 134 (2020).

[53] R.A. Tasca, J.R.L. Almeida, D.G. Silva, F.M. Melo e H.E. Toma, Química Nov. Na Esc. 37, 236 (2015).

[54] O. Domingues, L.K. Santos, R.D. Herculano, D.L. Flumignan e A.V. Paula, J. Basic Appl. Pharm. Sci. 39, 1 (2018).

[55] T. Leshuk, H. Krishnakumar e F. Gu, J. Nanosci. Nanotechnol. 15, 5378 (2015).

[56] M. Mahdavi, M. Bin Ahmad, M.J. Haron, F. Namvar, B. Nadi, M.Z.A. Rahman, J. Amin, Molecules 18, 7533 (2013)

[57] A.L. Drummond, N.C. Feitoza, G.C. Duarte, M.J.A. Sales, L.P. Silva, J.A. Chaker, A.F. Bakuzis e M.H. Sousa, J. Nanosci. Nanotechnol. 12, 8061 (2012).

[58] J. Lim, S.P. Yeap e H.X. Che, S.C. Nanoscale Res. Lett. 8, $381(2013)$.

[59] N.P. Silveira e F.C. Giacomelli, Tópicos Em Nanociência e Nanotecnologia (Ed. Da UFRGS, Porto Alegre, 2008), v. 1, p. 93.

[60] R. Pecora, Dynamic light scattering: applications of photon correlation spectroscopy (Springer Science \& Business Media, New York, 2013), v. 2, p. 85.

[61] E. Velichko, S. Makarov, E. Nepomnyashchaya e G. Dong, Biology (Basel). 9, 123 (2020).

[62] Y. Zhao, Z. Qiu e J. Huang, Chinese J. Chem. Eng. 16, 451 (2008).

[63] A. Zhu, L. Yuan e T. Liao, Int. J. Pharm. 350, 361 (2008).

[64] A. Zhu, L. Yuan e S. Dai, J. Phys. Chem. C. 112, 5432 (2008).

[65] L. He, M. Wang, J. Ge e Y. Yin, Acc. Chem. Res. 45, 1431 (2012).

[66] J.F. Lin, C.C. Tsai e M.Z. Lee, J. Magn. Magn. Mater. 372, 147 (2014).

[67] K. Wu, D. Su, J. Liu, R. Saha e J.P. Wang, Nanotechnology 30, 1 (2019).

[68] N.C.V. Rost, G.C. Gonçalves, D. Palma, M.L. Castilho, P.M.S.C.M. Leite, F.M. Broca, L. Raniero, Rev. Univap. 22, 872 (2017).

[69] R. Costo, V. Bello, T. González-Carreño, S. Veintemillas-Verdaguer e D. Wang, Mater. Res. Bull. 48, 4294 (2013).

[70] M. Aliahmad e N.N. Moghaddam, Mater. Sci. 31, 264 (2013).

[71] J. Drbohlavova, R. Hrdy, V. Adam, R. Kizek, O. Schneeweiss e J. Hubalek, Sensors. 9, 2352 (2009). 
[72] W. Lu, Y. Shen, A. Xie, W. Zhang, J. Magn. Magn. Mater. 322, 1828 (2010).

[73] L.M. Sikhwivhilu, S.S. Ray e N.J. Coville, Appl. Phys. A. 94, 963 (2009).

[74] D.P. Ausubel, Aquisição e retenção de conhecimentos: uma perspectiva cognitiva (Plátano, Lisboa, 2003), v. 1, p. 243.

[75] L.S. Vygotskii, A.R. Luriia, A.N. Leont'ev e J.C. Neto, Linguagem, desenvolvimento e aprendizagem (Ícone, São Paulo, 2005), v. 16, p. 232.

[76] L. Bardin, Análise de conteúdo (Edições 70, Lisboa, 2011), v. 1, p. 229.

[77] J.M.L. Oliveira, C.U. Ferreira e M. Almeida, em: Anais do XX Simpósio Nacional de Ensino de Física (São Paulo, 2013). 